\title{
Patient Outcomes in a Medicaid Managed Care Lock-In Program
}

\author{
Theresa R. F. Dreyer, MPH; Thomas Michalski, MBA, RPh; and Brent C. Williams, MD, MPH
}

\begin{abstract}
BACKGROUND: Prescription drug abuse is a growing epidemic in the United States, and opioids are among the most commonly abused and misused controlled substances. Managed care organizations can use pharmacy lock-in programs to limit patients' access to opioids by requiring that they receive all scripts from 1 prescriber, potentially reducing inappropriate use.

OBJECTIVE: To evaluate opioid use patterns among patients in a Medicaid managed care lock-in program limiting opioid coverage to prescriptions written by assigned prescribers.
\end{abstract}

METHODS: This retrospective cohort study included all patients enrolled in the lock-in program at Blue Care Network (BCN) of Michigan Medicaid managed care from March 2008 through May 2013, with outcomes assessed through August 2013. BCN medical and pharmacy claims, the Michigan Automated Prescription System, and Blue Cross Complete Controlled Substance Committee reports were used to assess outcomes at $6,12,24$, and 36 months after enrollment. Patients were defined as "stable" if they exclusively filled opioid prescriptions from assigned prescribers or received treatment for opioid dependence and "unstable" if they purchased prescription opioids with cash or submitted opioid claims not prescribed by assigned providers.

RESULTS: Of the 59 patients enrolled in the program, over half $(55.9 \%)$ dropped BCN coverage, and 1 died while enrolled. The proportion of patients who dropped coverage fell as time in the program increased, from $29 \%$ in the first 6 months to $11 \%$ semiannually after 24 months. Among those who remained enrolled, the proportion of stable patients increased from $31 \%$ at 6 months to $78 \%$ at 36 months. The small sample size did not permit formal statistical analysis.

CONCLUSIONS: The finding that most patients exited the program by dropping coverage was an unintended consequence meriting further investigation. Conversely, the finding that patients who remained enrolled largely achieved desired outcomes indicates that this program played an important role in addressing opioid abuse.

J Manag Care Spec Pharm. 2015;21(11):1006-12

Copyright $\odot 2015$, Academy of Managed Care Pharmacy. All rights reserved.

\section{What is already known about this subject}

Most previous evaluations of Medicaid lock-in programs focused on program costs, demonstrating reduced costs among patients enrolled in the programs, but they did not evaluate clinical outcomes.

The Centers for Medicare \& Medicaid Services recommends that all state Medicaid programs implement lock-in programs to curb opioid abuse and misuse.

\section{What this study adds}

This study demonstrated that the majority of patients enrolled in a Medicaid managed care lock-in program dropped Medicaid managed care coverage, but those who remained enrolled were more likely to achieve stable outcomes with increased time in the program.

The majority of patients who dropped coverage did so within 6 months of entering the lock-in program.

As length of time in the program increased, a larger proportion of patients decreased or stopped use of prescription opioids, maintained steady use under physician supervision, or entered maintenance replacement therapy.

$\mathrm{P}$ rescription drug abuse is a growing epidemic in the United States, with prescription drugs driving the rise in fatal drug overdoses over the past 2 decades. Opioids are among the most commonly abused and misused controlled substances. Opioids are a class of drugs that relieve pain and include morphine, oxycodone, methadone, hydrocodone, and other narcotic drugs. Nationwide, deaths from prescription opioid overdoses now exceed deaths from heroin and cocaine combined. ${ }^{1}$ From 1999 to 2008, the increased prevalence of opioid-related deaths and admissions paralleled the increase in prescribing opioids for pain management. ${ }^{1}$ Providers have been prescribing opioids more extensively for pain control since the 1990s in response to changing practice guidelines for pain management and rising concerns about untreated pain. The majority of opioid-related morbidity and mortality in the United States results from patients taking opioids as prescribed, rather than from abuse and misuse. ${ }^{2}$

From 2001 to 2008, overdose deaths, drug abuse treatment, and sales of prescription opioids increased considerably. In $2008,14,800$ people died of opioid overdoses, representing $73.8 \%$ of all deaths from prescription drug overdoses that year, nearly 4 times higher than the 1999 death rate. ${ }^{1}$ In 2011, opioids other than heroin represented approximately $10 \%$ of all substance abuse treatment admissions nationally, up from $2 \%$ in 2001. Among adolescents, opioids other than heroin rose from $29 \%$ of national admissions for substance abuse treatment in 2001 to $66 \%$ in 2011. ${ }^{3}$ Approximately 488,000 emergency department (ED) visits involved nonmedical use of opioids in 2011, accounting for 29\% of all ED visits related to prescription drug abuse and misuse. ED visits related to opioid abuse or misuse rose 183\% from 2004 to 2011, compared with 132\% 
for nonmedical use of pharmaceuticals overall. ${ }^{4}$ In Michigan, opioids other than heroin were the drug of choice for over 7,000 people admitted for substance abuse treatment in 2013, representing nearly $14 \%$ of all admissions that year. ${ }^{5}$

Effective strategies to curb opioid abuse and misuse could defray the cost of this epidemic to the U.S. health care system. The most recent economic analysis of prescription opioid abuse and misuse found that nonmedical use had a total economic burden of $\$ 53.4$ billion, driven primarily by lost productivity and criminal justice expenditures. ${ }^{6}$ Direct medical costs are also higher among opioid abusers because of higher use of health care services and prescription drugs, as well as higher prevalence of comorbid conditions. ${ }^{7}$

In response to this crisis, payers, providers, regulatory agencies, and manufacturers have adopted a variety of approaches to curb opioid abuse and misuse..$^{8-13}$ Payer-based approaches include formulary controls; provider-directed interventions such as education; feedback from prescription monitoring programs; screening and intervention programs; and Medicaid lock-in programs. ${ }^{8,9}$ Lock-in programs are a way to limit use by patients who are "doctor shopping," or seeking multiple opioid prescriptions from different providers within a short period of time, often filling these prescriptions at multiple pharmacies. Nationally, an estimated $0.3 \%$ to $0.7 \%$ of opioid purchasers are doctor shoppers..$^{14,15}$

Lock-in programs, also called patient review and restriction programs, limit payment for opioids to prescriptions from a single prescriber and/or pharmacy. They are designed to prevent dangerously uncoordinated care and eliminate reimbursement for inappropriate or fraudulent opioid prescriptions. Most states run lock-in programs for Medicaid beneficiaries, and internal evaluations have consistently demonstrated cost savings. ${ }^{16-19}$ The Centers for Medicare and Medicaid Services (CMS) recommends that all state Medicaid programs implement lock-in programs. ${ }^{20}$ Little is known, however, about the clinical outcomes of payer-based lock-in programs. The purpose of this study was to examine the opioid use patterns of patients enrolled in the Medicaid lock-in program of a large managed care organization for up to 36 months, to determine whether enrollment in the program was correlated with reductions in inappropriate use, such as uncoordinated prescribing and potential doctor-shopping behaviors.

\section{Methods}

\section{Study Population and Data}

Blue Care Network (BCN) is the Blue Cross Blue Shield of Michigan managed care organization, and its administrative arm for Medicaid patients was Blue Cross Complete (BCC) during the time of this study. This study used BCN medical and pharmacy claims data, supplemented by BCC Controlled Substance Committee reports, which identified patients eligible for the lock-in program and evaluated their behavior in the program quarterly. Data on cash purchases of prescription

\section{FIGURE 1 Patient Screening and Enrollment in the Medicaid Managed Care Lock-In Program}

Patients flagged on quarterly basis in BCC Controlled Substance Shoppers Report

(Criteria: $\geq 10$ controlled substance prescriptions from $\geq 4$ prescribers within a 3-month period)

Patients meeting criteria were reviewed by $\mathrm{BCN}$ pharmacist to determine if case should be evaluated by the BCC Controlled Substance Committee $(\mathrm{N}=484)$

No further action $(\mathrm{N}=380)$

Patients reviewed by the BCC Controlled Substance Committee at quarterly meetings $(\mathrm{N}=104)$

Follow-up next quarter

No further action $(\mathrm{N}=45)$
Patients enrolled in the Medicaid managed care lock-in program $(\mathrm{N}=59)$

$B C C=$ Blue Cross Complete; $B C N=$ Blue Care Network

opioids were drawn from the Michigan Automated Prescription System (MAPS). MAPS is the state's prescription drug monitoring program, a database of all controlled substance purchases in the state, which documents the patient's name, date of purchase, drug name and quantity, prescriber and pharmacy identification numbers, and method of payment. MAPS was reviewed quarterly for each patient in the lock-in program to determine whether they had obtained controlled substances outside of $\mathrm{BCN}$ Medicaid coverage by paying cash. The University of Michigan Institutional Review Board and BCN Internal Review and Confidentiality Committee reviewed this study and determined that it was exempt from ongoing review.

Patients were eligible for enrollment if they filled $\geq 10$ controlled substance prescriptions from $\geq 4$ prescribers within a 3 -month period. BCN chose this threshold because it may signal potential "doctor shopping" or dangerously uncoordinated care. The criteria were designed to avoid a large number of false positives but not to miss severe cases of abuse. 


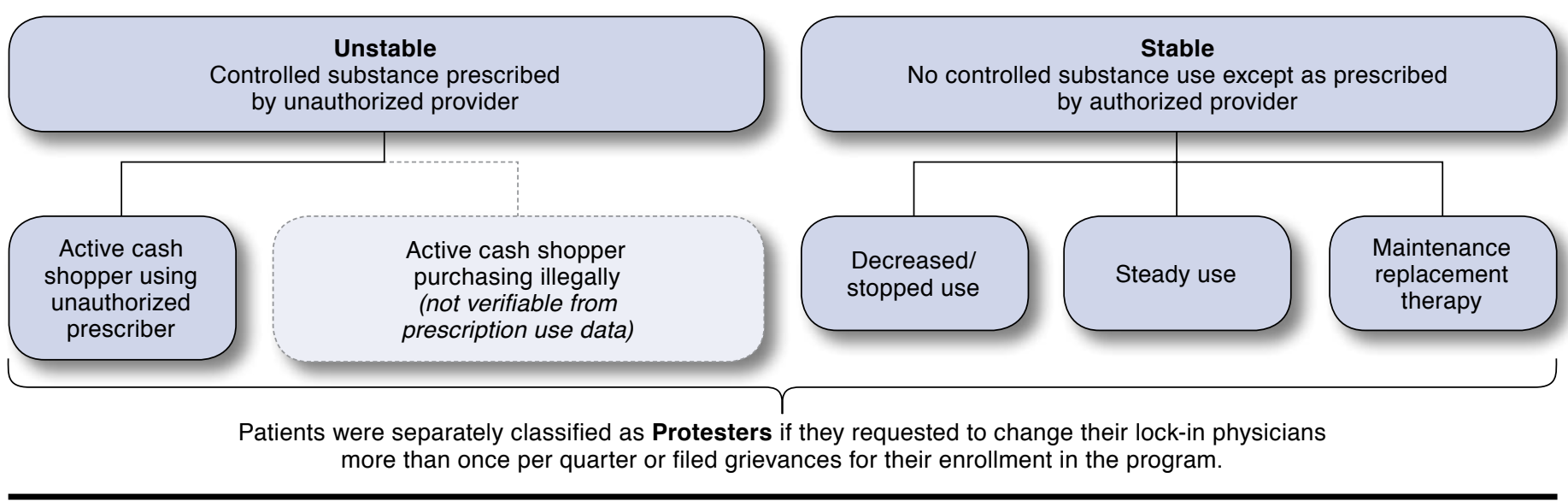

Patients meeting these criteria were flagged in BCC Controlled Substance reports on a quarterly basis and were reviewed by a $\mathrm{BCN}$ pharmacist to determine whether the BCC Controlled Substance Committee should evaluate the case (Figure 1). The BCC Controlled Substance Committee was composed of a BCN physician, pharmacist, and case manager, as well as a medical director and case manager from the University of Michigan Health System Complex Care Management Program. Pharmacy claims for controlled substances were defined as schedule II to V drugs, as classified by the U.S. Drug Enforcement Administration. Patients with diagnoses that could have justified the observed high use of controlled substances were not enrolled in the program, such as those with cancer, recent injury or surgery, chronic pain conditions, or neuralgias, but were flagged for follow-up if they appeared on subsequent reports. For the remaining patients, the committee contacted their primary care physicians (PCPs) to ask for their agreement to be sole opioid prescribers for these patients. Once the PCPs agreed, then the patients were informed via letter that only controlled substance prescriptions provided by the PCP would be covered.

\section{Study Design}

This retrospective cohort study describes the outcomes of all patients enrolled in the BCN lock-in program from March 2008 through May 2013. Patient outcomes were evaluated at 6 , 12,24 , and 36 months after the date of enrollment in the program. Patients were followed through August 31, 2013, or until 1 of the following events occurred: completion of 36 months in the program, termination of BCN Managed Care coverage, death (recorded in the quarterly BCC Controlled Substance Committee reports), or loss to follow-up (i.e., no longer appear- ing in the reports). No comparable control group was available for this population because all patients who met the lock-in program criteria were enrolled.

Patient opioid use was broadly categorized as "stable" or "unstable" at each time interval, based on BCC Controlled Substance Committee reports. For the purposes of this study, patients were assumed to use any opioid prescriptions they filled. Stable use was defined as opioid use only as prescribed by the assigned PCP (Figure 2). Patients with stable use decreased or stopped using prescription opioids, maintained steady use, or entered maintenance replacement therapy. Patients who maintained steady use of opioids used the drugs prescribed by the lock-in provider but did not obtain controlled substances through other providers. Patients who entered maintenance therapy were identified because they received prescriptions to treat opioid abuse (e.g., buprenorphine), had medical claims for treatment, or their lock-in providers directly communicated this information to $\mathrm{BCN}$ staff. In contrast, unstable use was defined as attempted or actual use of prescription opioids not prescribed by the assigned PCP. Patients with unstable outcomes either submitted claims for opioids written by other providers or paid cash for opioids prescribed by other providers.

Patients were independently classified as "protesters" in each time interval if they sought to circumvent the lock-in program. Patients were considered protesters if they requested to change their lock-in PCPs or called BCN Member Services more than once per quarter or if they filed grievances for their enrollment in the program. In some cases, patients may have sought to change their lock-in PCP for legitimate reasons, including dissatisfaction with the services they received or provider retirement. However, patients were not classified as protesters for seeking to change their lock-in PCP less frequently than once per quarter. 


\begin{tabular}{ll}
\hline \multicolumn{1}{c|}{ TABLE 1) } & $\begin{array}{l}\text { Characteristics of Patients Enrolled } \\
\text { in the Medicaid Managed Care } \\
\text { Lock-In Program }\end{array}$ \\
\hline Median age & 37 \\
\hline Age range & $22-64$ \\
\hline \% female & 72.9 \\
\hline Average number of diagnoses per patient & 1.2 \\
\hline
\end{tabular}

\section{Results}

During the study period, 59 BCN Medicaid managed care patients were enrolled in the lock-in program. Enrolled patients had a median age of 37 years (range 22-64), were $72.9 \%$ female, and had an average of 1.2 chronic conditions (Table 1). The most common chronic conditions were migraine (20 patients), low back pain (17), and lumbago (13). Two patients had previously been diagnosed with drug withdrawal, and 1 was diagnosed with a mental health condition.

Endpoints experienced by patients enrolled in the Medicaid managed care lock-in program were distributed as follows: over half (54\%) of the 59 enrollees dropped BCN coverage, and 1 patient (2\%) died 1 month after enrollment in the program (Table 2). All the remaining patients were followed through August 31, 2013 (22 patients, 37\%) or for 36 months (4 patients, 7\%). No patients were lost to follow-up.

As time in the program increased, the proportion of patients who dropped coverage decreased. Within 6 months of enrollment, $29 \%$ of the 59 patients who entered the lock-in program had terminated BCN coverage, while an additional 2\% (1 patient) had died (Table 3 and Figure 3). Between 6 and 11 months, the proportion of patients who left declined to $18 \%$ of the remaining patients. From 12 to 23 months (double the length of the previous time intervals), $25 \%$ of the patients dropped BCN coverage, averaging approximately 13\% each 6 months. The attrition rate between 24 and 36 months after enrollment was $22 \%$, or $11 \%$ semiannually.

However, the proportion of patients with stable outcomes increased as time in the program increased. Six months after enrollment, only 18 of the original 59 patients (31\%) remained enrolled and were stable. By comparison, 22 (37\%) patients remained enrolled but were unstable because of cash purchases of prescription opioids or attempts to bill $\mathrm{BCN}$ for opioid prescriptions not written by their assigned provider. At 12 months, the proportion of stable patients rose to 50\% (19 of the 38 patients enrolled for that duration), while the proportion of unstable patients declined to $26 \%$ (10 patients). The proportion of stable patients continued to increase with time in the program, to $58 \%$ at 24 months (14 of 24 patients) and $78 \%$ at 36 months ( 7 of 9 patients). Within each time interval, most stable patients decreased their use of prescription opioids or stopped use entirely.

\section{TABLE 2 \\ Endpoints Experienced by Patients Enrolled in the Medicaid Managed Care Lock-In Program}

\begin{tabular}{l|rc}
\hline & Number of Patients (\%) \\
\hline Terminated BCN managed care coverage & 32 & $(54.2)$ \\
\hline $\begin{array}{l}\text { Remained enrolled through the end of the study } \\
\text { period (August 31, 2013) }\end{array}$ & 22 & $(37.3)$ \\
\hline Completed 36 months in the program & 4 & $(6.8)$ \\
\hline Died & 1 & $(1.7)$ \\
\hline Lost to follow-up & 0 & $(0.0)$ \\
\hline Total & 59 & $(100.0)$ \\
\hline$B C N=$ Blue Care Network. & & \\
\hline
\end{tabular}

Few patients protested their enrollment in the lock-in program after remaining in the program for over 6 months. At 6 months, $19 \%$ of the patients protested their enrollment in the program. This proportion fell to 3\% at 12 months after enrollment, rose slightly to $4 \%$ at 24 months (double the length of the previous time interval), and declined to zero at 36 months post-index.

\section{Discussion}

In this evaluation of a payer-based lock-in program to curb opioid abuse in a Medicaid managed care program, patient outcomes were strongly related to the amount of time spent in the program. In the early months after enrollment, the most common outcome was disenrollment from the managed care program. Patients may have switched to a different Medicaid managed care plan, entered Medicaid fee-for-service, acquired private coverage, or become uninsured.

The attrition rate for the study population was higher than that of the general population in each time interval, particularly within 6 months of enrollment in the program. General attrition rates were calculated using a cross-section of all individuals enrolled in BCC on March 1, 2010. Approximately $20 \%$ of the total population dropped BCC coverage within 6 months of this date, as did an additional 12\% between 6 to 11 months. From 12 to 23 months, 18\% dropped BCC coverage ( $9 \%$ semiannually), and 15\% dropped coverage from 24 to 36 months ( $7 \%$ semiannually).

Although attrition rates were high for patients enrolled in the lock-in program, the patients who remained in the program were more likely to demonstrate improved patterns of opioid use-cessation, reduction, steady use, or a switch to maintenance replacement therapy. Cessation and decreased use were positively correlated with increased time in the program. Longer periods in the program also corresponded with decreased likelihood of leaving Medicaid managed care coverage or of maintaining coverage while protesting enrollment in the program. Among patients who remained in the program, the lock-in program may have succeeded in its goal of eliminating uncoordinated opioid prescribing among providers. 
TABLE 3 Patient Outcomes in the Medicaid Managed Care Lock-In Program over Time

\begin{tabular}{|c|c|c|c|c|c|c|c|c|}
\hline & \multicolumn{8}{|c|}{ Observation Interval (Months After Enrollment) } \\
\hline & \multicolumn{2}{|c|}{$0-6$} & \multicolumn{2}{|c|}{$6-12$} & \multicolumn{2}{|c|}{$12-24$} & \multicolumn{2}{|c|}{$24-36$} \\
\hline Patients entering observation period, $\mathrm{n}$ & \multicolumn{2}{|c|}{59} & \multicolumn{2}{|c|}{38} & \multicolumn{2}{|c|}{24} & \multicolumn{2}{|r|}{9} \\
\hline \multicolumn{9}{|c|}{ Outcomes during observation period among patients not completing the follow-up period, $n$ (\%) } \\
\hline Terminated & 17 & $(28.8)$ & 7 & $(18.4)$ & 6 & 25.0) & 2 & $22.2 \%$ \\
\hline Deceased & 1 & $(1.7)$ & \multicolumn{2}{|c|}{ - } & \multicolumn{2}{|c|}{ - } & \multicolumn{2}{|r|}{-} \\
\hline \multicolumn{9}{|c|}{ Use status at end of observation period, ${ }^{a} \mathbf{n}(\%)$} \\
\hline Unstable use & 22 & $(37.3)$ & 10 & $(26.3)$ & 3 & $(12.5)$ & 0 & $(0.0)$ \\
\hline Stable use & 18 & $(30.5)$ & & $(50.0)$ & 14 & $(58.3)$ & 7 & $(77.8)$ \\
\hline Decrease/stop & \multicolumn{2}{|c|}{10} & \multicolumn{2}{|c|}{13} & \multicolumn{2}{|c|}{7} & \multicolumn{2}{|r|}{6} \\
\hline Steady use & \multicolumn{2}{|c|}{5} & \multicolumn{2}{|c|}{5} & \multicolumn{2}{|c|}{6} & \multicolumn{2}{|r|}{0} \\
\hline Maintenance replacement therapy & \multicolumn{2}{|c|}{3} & \multicolumn{2}{|c|}{1} & \multicolumn{2}{|c|}{1} & \multicolumn{2}{|r|}{1} \\
\hline Unknown & 1 & $(1.7)$ & 2 & $(5.3)$ & 1 & $(4.2)$ & & - \\
\hline
\end{tabular}

Most previous evaluations of lock-in programs have focused on program costs, demonstrating reduced costs among patients enrolled in the programs, and few of these evaluations were published in peer-reviewed journals. ${ }^{21}$ For example, Oklahoma's SoonerCare pharmacy lock-in program reported $\$ 31,500$ in savings in reduced opioid prescriptions in 2009, averaging $\$ 600$ per patient, without impacting patients' use of maintenance medications. ${ }^{18,19}$ One previous article outlined payer-based interventions to address opioid abuse, and another assessed an intervention in which a payer supplied providers with information on specific patients with problematic opioid use. ${ }^{8,13} \mathrm{~A}$ third article evaluated pharmacists' experiences in implementing the Medicaid lock-in program in North Carolina. ${ }^{22}$

To our knowledge, this is the first evaluation of opioid use patterns and participation over time among patients in a lockin program in the academic literature. The prevalence of back pain and headaches in our study population aligns with a 2010 study on opioid trends and risks, which found that individuals with these chronic conditions were more likely to abuse or become dependent on opioids, as were individuals with prior mental health and substance abuse diagnoses. The same study found that individuals aged between 31 and 40 years were at even higher risk for opioid abuse or misuse than those with prior mental health or substance abuse diagnoses. ${ }^{23}$

\section{Limitations}

This study has several limitations, including the small numbers of lock-in program enrollees, the lack of a control group, and inclusion of patients from a single Medicaid managed care organization. For these reasons, the study's findings may not be generalizable to other lock-in programs. While the lack of a control group prevents direct comparison of our patients' propensity to leave the managed care program with other patients, the attrition rate after enrollment in the program exceeds that of the
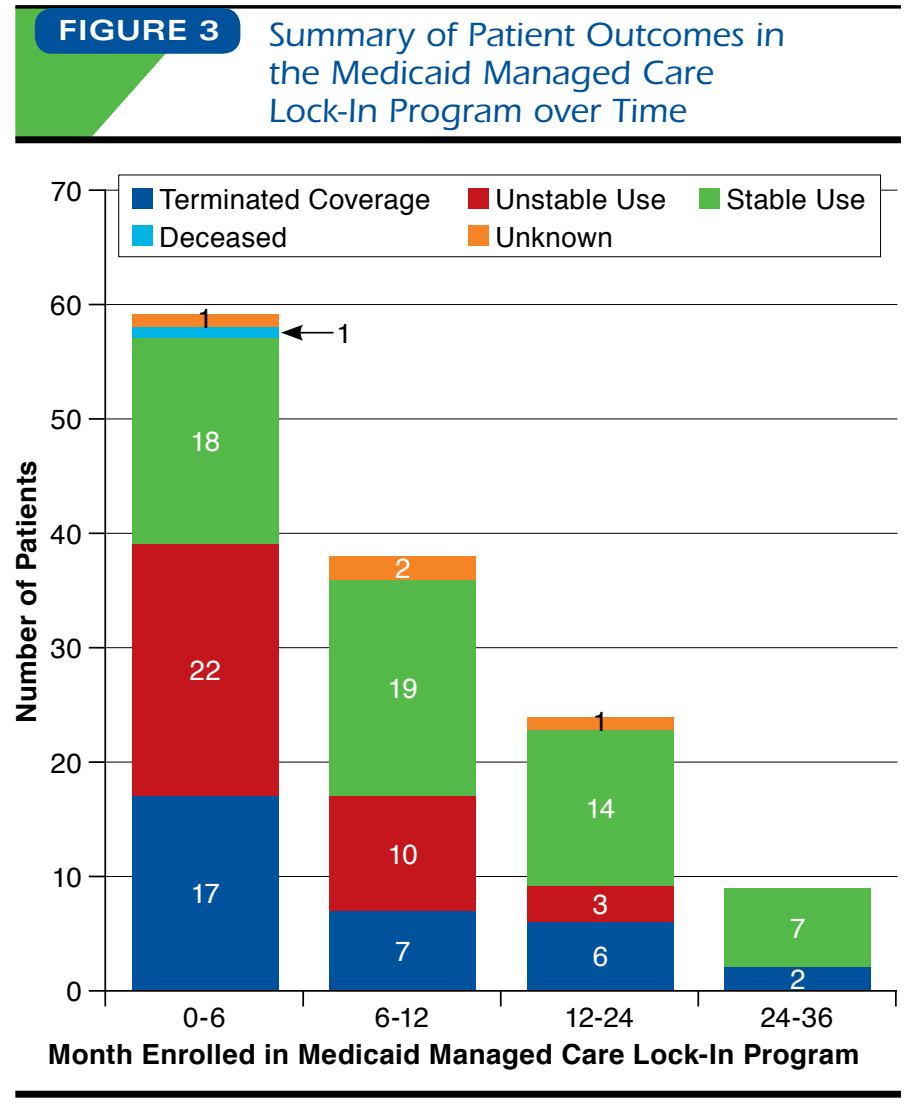

general population. Patients who were more likely to continue unstable opioid use may also have been more likely to terminate BCC coverage in order to leave the lock-in program. Conversely, patients who were more likely to adopt more appropriate opioid use may have been more likely to remain enrolled. This is a major confounding factor in the correlation between patients' 
time in the program and decision to terminate coverage because patients who remained enrolled over time may have been those least likely to terminate coverage to begin with.

Additional limitations include the lack of data on prescription drug use outside Michigan or illegal purchases of controlled substances, which could have resulted in misclassification of some individuals as having improved opioid use patterns. Additionally, our dataset did not include medications delivered during hospitalization or ED visits, which may also have undercounted some patients' opioid use.

\section{Conclusions}

This study is the first to examine patient outcomes in a lock-in program for opioids, adding to the evidence on payer-based interventions to address prescription drug misuse and abuse. The apparent effect of promoting exit from managed care by patients early in enrollment in the lock-in program, if verified in future studies, is an unintended consequence of the program that merits investigation. Conversely, the apparent achievement of desired outcomes among patients who remained in the program suggests that lock-in programs play an important, albeit limited, role in addressing the opioid abuse epidemic. Our findings support the CMS recommendation that all state Medicaid programs implement lock-in programs. ${ }^{20}$ In addition to this payer-based approach, other approaches to opioid abuse should continue, including provider-directed programs to improve physician prescribing and clinical practices, ${ }^{2,8,13}$ and regulatory approaches controlling prescribing practices and reporting requirements. ${ }^{8-12}$

\section{Authors}

THERESA R. F. DREYER, MPH, is Healthcare Analyst, Center for Healthcare Research $\&$ Transformation, University of Michigan, Ann Arbor; THOMAS MICHALSKI, MBA, RPh, is Clinical Pharmacist, Blue Care Network of Michigan, Southfield, MI; and BRENT C. WILLIAMS, MD, MPH, is Associate Professor of Internal Medicine, University of Michigan Medical School, and Medical Director, Complex Care Management Program, University of Michigan Health System, Ann Arbor.

AUTHOR CORRESPONDENCE: Theresa R. F. Dreyer, MPH, 2929 Plymouth Rd., Ste. 245, Ann Arbor, MI 48105. Tel.: 734.998.8517; Fax: 734.998.8515; E-mail: tdreyer@med.umich.edu.

\section{DISCLOSURES}

This study received no external funding. Michalski is a Blue Care Network employee and did not receive additional compensation for work on this study. There are no other potiential conflicts of interest.
Study concept and design were contributed primarily by Williams, with assistance from Dreyer and Michalski. Michalski took the lead in data collection, assisted by Williams, with data interpretation performed by Dreyer and Michalski. The manuscript was written and revised primarily by Dreyer, along with Williams and with assistance from Michalski.

\section{REFERENCES}

1. Centers for Disease Control and Prevention. Vital signs: overdoses of prescription opioid pain relievers-United States, 1999-2008. MMWR Morb Mortal Wkly Rep. 2011;60(43):1487-92. Available at: http://www.cdc.gov/ mmwr/preview/mmwrhtml/mm6043a4.htm. Accessed September 15, 2015

2. Manchikanti L, Helm S 2nd, Fellows B, et al. Opioid epidemic in the United States. Pain Physician. 2012;15(3 Suppl):ES9-38.

3. Substance Abuse and Mental Health Services Administration, Center for Behavioral Health Statistics and Quality. Treatment Episode Data Set (TEDS): 2001-2011. National admissions to substance abuse treatment services. BHSIS Series S-65, HHS Publication No. (SMA) 13-4772. Rockville, MD: Substance Abuse and Mental Health Services Administration, 2013. Available at: http://www.samhsa.gov/data/2k13/TEDS2011/TEDS2011N.pdf. Accessed September 15, 2015.

4. Substance Abuse and Mental Health Services Administration. Drug abuse warning network, 2011: national estimates of drug-related emergency department visits. HHS Publication No. (SMA) 13-4760, DAWN Series D-39. Rockville, MD: Substance Abuse and Mental Health Services Administration, 2013. Available at: http://www.samhsa.gov/data/sites/ default/files/DAWN2k11ED/DAWN2k11ED/DAWN2k11ED.htm. Accessed September 15, 2015.

5. Substance Abuse and Mental Health Services Administration. Table: Substance abuse treatment admissions by primary substance of abuse, according to sex, age group, race, and ethnicity. Michigan, 2013. In: Substance Abuse and Mental Health Services Administration, Center for Behavioral Health Statistics and Quality. Treatment Episode Data Set (TEDS). Updated July 6, 2015. Available at: http://wwwdasis.samhsa.gov/ webt/quicklink/MI13.htm. Accessed September 15, 2015.

6. Hansen RN, Oster G, Edelsberg J, et al. Economic costs of nonmedical use of prescription opioids. Clin J Pain. 2011;27(3):194-202

7. White AG, Birnbaum HG, Mareva MN, et al. Direct cost of opioid abuse in an insured population in the United States. J Manag Care Pharm. 2005;11(6):469-79. Available at: http://www.amcp.org/data/jmcp/3.pdf.

8. Katz NP, Birnbaum H, Brennan MJ, et al. Prescription opioid abuse: challenges and opportunities for payers. Am J Manag Care. 2013;19(4):295-302.

9. Sehgal N, Manchikanti L, Smith HS. Prescription opioid abuse in chronic pain: a review of opioid abuse predictors and strategies to curb opioid abuse. Pain Physician. 2012;15(3 Suppl):ES67-92

10. U.S. Food and Drug Administration. Risk evaluation and mitigation strategy (REMS) for extended-release and long-acting opioids. Updated July 13, 2015. Available at: http://www.fda.gov/drugs/drugsafety/informationbydrugclass/ucm 163647.htm. Accessed September 15, 2015.

11. Meier B. F.D.A. urging a tighter rein on painkillers. The New York Times October 24, 2013. Available at: http://www.nytimes.com/2013/10/25/business/fda-seeks-tighter-control-on-prescriptions-for-class-of-painkillers. html?pagewanted=all. Accessed September 15, 2015

12. Strassels SA. Economic burden of prescription opioid misuse and abuse. Am J Manag Care. 2009;15(7):556-62.

13. Gonzalez AM, Kolbasovsky A. Impact of a managed controlled-opioid prescription monitoring program on care coordination. Am J Manag Care. 2012;18(9):516-24.

14. McDonald DC, Carlson KE. Estimating the prevalence of opioid diversion by "doctor shoppers" in the United States. PloS One. 2013;8(7):e69241. 
15. Cepeda MS, Fife D, Chow W, et al. Opioid shopping behavior: how often, how soon, which drugs, and what payment method. J Clin Pharmacol. 2013;53(1):112-17.

16. Melnikow J, Yang Z, Soulsby M, et al. Approaches to Drug Overdose Prevention Analytical Tool (ADOPT): evaluating cost and health impacts of a Medicaid patient review \& restriction program. Center for Healthcare Policy and Research. December 2012. Available at: http://www.ucdmc.ucdavis. edu/iphi/Programs/OOD/resources/CDC\%20Opioid\%20Project_Final\%20 Report.pdf. Accessed September 15, 2015.

17. Best S. PMP use by Medicaid. December 3, 2012. Available at: http://www. pdmpexcellence.org/sites/all/pdfs/Best.pdf. Accessed September 15, 2015.

18. Oklahoma Health Care Authority. News Release. SoonerCare pharmacy lock-in program promotes appropriate use of medications. September 9 , 2009. Available at: https://www.okhca.org/about.aspx?id=10973. Accessed September 15, 2015.

19. Mitchel L. Pharmacy lock-in program promotes appropriate use of resources. J Okla State Med Assoc. 2009;102(8):276.
20. Centers for Medicare \& Medicaid Services. Drug diversion in the Medicaid program: state strategies for reducing prescription drug diversion in Medicaid. January 2012. Available at: http://www.cms.gov/MedicareMedicaid-Coordination/Fraud-Prevention/MedicaidIntegrityProgram/downloads/drugdiversion.pdf. Accessed September 15, 2015.

21. Roberts AW, Cockrell A. Assessing the present state and potential of Medicaid controlled substance lock-in programs. Am J Manag Care. 2014;20(5):439-46.

22. Werth SR, Sachdeva N, Roberts AW, et al. North Carolina Medicaid recipient management lock-in program: the pharmacist's perspective. J Manag Care Spec Pharm. 2014;20(11):1122-28. Available at: http://www. amcp.org/JMCP/2014/November/18727/1033.html.

23. Edlund MJ, Martin BC, Fan M, et al. Risks for opioid abuse and dependence among recipients of chronic opioid therapy: results from the TROUP study. Drug Alcohol Depend. 2010;112(1-2):90-98. 